\title{
Interval Valued Fuzzy Ideals of Near-rings and its Anti- homomorphism
}

\author{
V. Vetrivel, P. Murugadas ${ }^{\dagger}$ \\ * Department of Mathematics, Annamalai University, \\ Annamalainagar-608002, India. \\ $\dagger$ Department of Mathematics, Govt. Arts and Science College, \\ Karur-639005, India.
}

\begin{abstract}
Aim of this study is to investigate anti-homomorphic images and preimages of semiprime and primary ideals in interval valued fuzzy Near-rings. Further some results on f-invariant interval valued fuzzy ideal, f-invariant strongly primary interval valued fuzzy ideal and f-invariant semiprime interval valued fuzzy ideals of Near-rings are discussed.
\end{abstract}

2010 AMS Classification: 03E72, 08A72 and 13A15

Keywords: Semiprime, primary ideal and f-invariant.

\section{Introduction}

The notion of a fuzzy set was introduced by Zadeh [13] in 1965, utilizing which Rosenfeld [11] has defined fuzzy subgroups. In 1975, Zadeh [16] investigated the notion of interval valued fuzzy subsets (in short i-v fuzzy subsets) where the values of the membership functions are closed intervals of numbers instead of single numbers. Liu introduced the concept of a fuzzy ideal of a near-ring in [8]. The concepts of prime fuzzy ideals, primary fuzzy ideals for ring were introduced in [9]. In 1991, Abou-Zaid [1] also exposed some results in fuzzy subnear-rings and fuzzy ideals in

*vetrivelmath@gmail.com

†bodi_muruga@yahoo.com 
near-rings. Jun and Kim [4] and Davvaz [5] applied a few concepts of interval valued fuzzy subsets in near-rings. Sheikabdullah and Jeyaraman has discussed anti-homomorphic images and pre-images of prime fuzzy ideals and anti-homomorphic image of primary fuzzy ideals in a ring in [13].

The aim of this paper is to define and study i-v fuzzy primary ideals of a near ring $N$ and investigate anti-homomorphic images and pre-images of semi-prime, strongly primary i-v fuzzy ideals.

\section{Preliminaries}

Definition 2.1. [15] A non-empty set $N$ with two binary operations + and . is called a near-ring if:

i. $(N,+)$ is a group

ii. $(N,$.$) is a semigroup$

iii. $x .(y+z)=x . y+x . z$ for all $x, y, z \in N$.

We will use the word Near-ring to mean left near-ring.

Definition 2.2. [15] Let $X$ be a non-empty universal set. A fuzzy subset $\mu$ of $X$ is a function $\mu: X \rightarrow[0,1]$.

Example 2.3. Let $N=\{a, b, c, d\}$ be the Klein's four group. Define addition and multiplication in $N$ as follows.

\begin{tabular}{|c|c|c|c|c|}
\hline+ & $a$ & $b$ & $c$ & $d$ \\
\hline$a$ & $a$ & $b$ & $c$ & $d$ \\
\hline$b$ & $b$ & $a$ & $d$ & $c$ \\
\hline$c$ & $c$ & $d$ & $b$ & $a$ \\
\hline$d$ & $d$ & $c$ & $a$ & $b$ \\
\hline
\end{tabular}

\begin{tabular}{|c|c|c|c|c|}
\hline$\cdot$ & $a$ & $b$ & $c$ & $d$ \\
\hline$a$ & $a$ & $a$ & $a$ & $a$ \\
\hline$b$ & $a$ & $a$ & $a$ & $a$ \\
\hline$c$ & $a$ & $a$ & $a$ & $a$ \\
\hline$d$ & $a$ & $a$ & $b$ & $b$ \\
\hline
\end{tabular}

Here $(N,+,$.$) is a left near-ring. Define an interval valued fuzzy subset \bar{\mu}: N \rightarrow$ $D[0,1]$ by $\bar{\mu}(a)=[0.7,0.8], \bar{\mu}(b)=[0.5,0.6], \bar{\mu}(c)=[0.3,0.4]=\bar{\mu}(d)$.

It can be verified that $\bar{\mu}$ is an $i-v$ fuzzy ideal of $N$.

Definition 2.4. [15] An interval number $\bar{a}$ on $[0,1]$ is a closed subinterval of $[0,1]$, that is, $\bar{a}=\left[a^{-}, a^{+}\right]$such that $0 \leq a^{-} \leq a^{+} \leq 1$ where $a^{-}$and $a^{+}$are the lower and upper end limits of $\bar{a}$ respectively. The set of all closed subintervals of $[0,1]$ is 
denoted by $D[0,1]$. We also identify the interval $[a, a]$ by the number $a \in[0,1]$. For any interval numbers $\bar{a}_{i}=\left[a_{i}^{-}, a_{i}^{+}\right], \bar{b}_{i}=\left[b_{i}^{-}, b_{i}^{+}\right] \in D[0,1], i \in I$, we define

$\max ^{i}\left\{\bar{a}_{i}, \bar{b}_{i}\right\}=\left[\max ^{i}\left\{a_{i}^{-}, b_{i}^{-}\right\}, \max ^{i}\left\{a_{i}^{+}, b_{i}^{+}\right\}\right]$,

$\min ^{i}\left\{\bar{a}_{i}, \bar{b}_{i}\right\}=\left[\min ^{i}\left\{a_{i}^{-}, b_{i}^{-}\right\}, \min ^{i}\left\{a_{i}^{+}, b_{i}^{+}\right\}\right]$,

inf $f^{i} \bar{a}_{i}=\left[\bigcap_{i \in I} a_{i}^{-}, \bigcap_{i \in I} a_{i}^{+}\right], \sup ^{i} \bar{a}_{i}=\left[\bigcup_{i \in I} a_{i}^{-}, \bigcup_{i \in I} a_{i}^{+}\right]$

In this notation $\overline{0}=[0,0]$ and $\overline{1}=[1,1]$. For any interval numbers $\bar{a}=$ $\left[a^{-}, a^{+}\right]$and $\bar{b}=\left[b^{-}, b^{+}\right]$on $[0,1]$, define

(1) $\bar{a} \leq \bar{b}$ if and only if $a^{-} \leq b^{-}$and $a^{+} \leq b^{+}$.

(2) $\bar{a}=\bar{b}$ if and only if $a^{-}=b^{-}$and $a^{+}=b^{+}$.

(3) $\bar{a}<\bar{b}$ if and only if $\bar{a} \leq \bar{b}$ and $\bar{a} \neq \bar{b}$

(4) $k \bar{a}=\left[k a^{-}, k a^{+}\right]$, whenever $0 \leq k \leq 1$.

Definition 2.5. [15] Let $X$ be any set. A mapping $\bar{A}: X \rightarrow D[0,1]$ is called an interval-valued fuzzy subset (briefly, $i$ - $v$ fuzzy subset) of $X$ where $D[0,1]$ denotes the family of all closed subintervals of $[0,1]$ and $\bar{A}(x)=\left[A^{-}(x), A^{+}(x)\right]$ for all $x \in X$, where $A^{-}$and $A^{+}$are fuzzy subsets of $X$ such that $A^{-}(x) \leq A^{+}(x)$ for all $x \in X$.

Note that $\bar{A}(x)$ is an interval (a closed subset of $[0,1]$ ) and not a number from the interval $[0,1]$ as in the case of fuzzy subset.

Definition 2.6. [15] A mapping min ${ }^{i}: D[0,1] \times D[0,1] \rightarrow D[0,1]$ defined by $\min ^{i}(\bar{a}, \bar{b})=\left[\min \left\{a^{-}, b^{-}\right\}, \min \left\{a^{+}, b^{+}\right\}\right]$for all $\bar{a}, \bar{b} \in D[0,1]$ is called an interval min-norm.

Definition 2.7. [15] A mapping $\max ^{i}: D[0,1] \times D[0,1] \rightarrow D[0,1]$ defined by $\max ^{i}(\bar{a}, \bar{b})=\left[\max \left\{a^{-}, b^{-}\right\}, \max \left\{a^{+}, b^{+}\right\}\right]$for all $\bar{a}, \bar{b} \in D[0,1]$ is called an interval max-norm.

Definition 2.8. [15] Let $N$ be a near-ring. An i-v fuzzy set $\mu$ of $N$ is called an $i-v$ fuzzy subnear-ring of $N$ if for all $x, y \in N$,

(i) $\bar{\mu}(x-y) \geq \min ^{i}\{\bar{\mu}(x), \bar{\mu}(y)\}$,

(ii) $\bar{\mu}(x y) \geq \min ^{i}\{\bar{\mu}(x), \bar{\mu}(y)\}$.

Definition 2.9. [15] An $i-v$ fuzzy subset $\bar{\mu}$ of a Near-ring $N$ is called an $i$-v fuzzy ideal of $N$ if $\bar{\mu}$ is an $i$-v fuzzy sub near-ring of $N$ and

(i) $\bar{\mu}(x)=\bar{\mu}(y+x-y)$

(ii) $\bar{\mu}(x y) \geq \bar{\mu}(y)$

(iii) $\bar{\mu}((x+i) y-x y) \geq \mu(i)$ for any $x, y, i \in N$ 
Proposition 2.10. [15] The anti-homomorphic image of an $i$-v fuzzy ideal of $N$ is an $i-v$ fuzzy ideal of $N^{\prime}$.

Proposition 2.11. [15] The homomorphic pre-image of an i-v fuzzy ideal of $N^{\prime}$ is an $i$ - $v$ fuzzy ideal of $N$.

\section{Main Results}

Definition 3.1. An $i-v$ fuzzy ideal $\bar{\mu}$ of a near-ring $N$ is called an $i-v$ prime fuzzy ideal if for any two $i-v$ fuzzy ideals $\bar{\sigma}$ and $\bar{\theta}$ of $N$ the condition $\bar{\sigma} \bar{\theta} \subseteq \bar{\mu}$ implies that $\bar{\sigma} \subseteq \bar{\mu}$ or $\bar{\theta} \subseteq \bar{\mu}$.

Definition 3.2. For an $i-v$ fuzzy ideal $\bar{\mu}$ of a near-ring, the $i$ - $v$ fuzzy radical of $\bar{\mu}$, denoted by $\sqrt{\bar{\mu}}$, is defined by $\sqrt{\bar{\mu}}=\cap\{\bar{\sigma}: \bar{\sigma}$ is an $i$-v fuzzy prime ideal of $N, \bar{\sigma} \subseteq$ $\left.\bar{\mu}, \bar{\sigma}_{*} \subseteq \bar{\mu}_{*}\right\}$. We denote $\bar{\mu}_{*}=\{x \in N: \bar{\mu}(x)=\bar{\mu}(0)\}$

Definition 3.3. An $i-v$ fuzzy ideal $\bar{\mu}$ of a near-ring $N$ is known as $i$ - $v$ fuzzy primary ideal if $\bar{\sigma} \bar{\theta} \subseteq \bar{\mu}$, then either $\bar{\sigma} \subseteq \bar{\mu}$ or $\bar{\theta} \subseteq \sqrt{\bar{\mu}}$.

Definition 3.4. An $i-v$ fuzzy ideal $\bar{\mu}$ if a near-ring $N$ is called $i$ - $v$ strongly primary fuzzy ideal of a near-ring $N$ if $\bar{\mu}$ is an i-v primary fuzzy ideal and $(\sqrt{\bar{\mu}})^{n} \subseteq \bar{\mu}$ for some $n \in N$.

Definition 3.5. An $i-v$ fuzzy ideal $\bar{\mu}$ of a near-ring $N$ is called $i$-v semi-prime if for any $i$-v fuzzy ideal $\bar{\sigma}$ of $N, \bar{\sigma}^{2} \subseteq \bar{\mu}$, then $\bar{\sigma} \subseteq \bar{\mu}$.

Definition 3.6. Let $X$ and $Y$ be two non-empty sets, $f: X \rightarrow Y, \bar{\mu}$ and $\bar{\sigma}$ be an $i$ - $v$ fuzzy subsets of $X$ and $Y$ respectively then $f(\bar{\mu})$, the image of $\bar{\mu}$ under $f$ is an $i$ - $v$ fuzzy subset of $Y$ denoted by

$$
f(\bar{\mu})(y)= \begin{cases}\sup ^{i}(x): f(x)=y & \text { if } f^{-1}(y) \neq \phi \\ 0 & \text { if } f^{-1}(y)=\phi .\end{cases}
$$

And $f^{-1}(\bar{\sigma})$, the pre-image of $\bar{\sigma}$ under $f$ is an $i$ - $v$ fuzzy subset of $X$ defined by $f^{-1}(\bar{\sigma})(x)=\bar{\sigma}(f(x)) \forall x \in X$.

Definition 3.7. If $\bar{\lambda}$ is an $i$-v fuzzy subset of $N$, then $\bar{\lambda}$ is said to have the sup property if for every subset $Y$ of $N$, there exists $y_{0} \in Y$ such that $\bar{\lambda}\left(y_{0}\right)=\{\bar{\lambda}(y) \mid y \in Y\}$. 
Definition 3.8. Let I be a non-empty $i$ - $v$ fuzzy subset of $N$. Define a function $\bar{C}_{I}$ : $N \rightarrow D[0,1]$ by

$$
\bar{C}_{I}(x)=\left\{\begin{array}{lc}
\overline{1} & \text { if } x \in I, \\
\overline{0} & \text { otherwise }
\end{array}\right.
$$

for all $x \in N$. Clearly $\bar{C}_{I}$ is an $i$-v fuzzy subset of $N . \bar{C}_{I}$ is called the $i$ - $v$ characteristic function of $I$. If the replace $I$ by $N, \bar{C}_{N}$ is the $i$-v characteristic function of $N$.

Definition 3.9. Let $N$ and $N^{\prime}$ be two near-rings, a mapping $f: N \rightarrow N^{\prime}$ is called an $i$-v fuzzy homomorphism if $f(\bar{\mu}+\bar{\sigma})=f(\bar{\mu})+f(\bar{\sigma})$ and $f(\bar{\mu} \bar{\sigma})=f(\bar{\mu}) f(\bar{\sigma})$ where $\bar{\mu}$ and $\bar{\sigma}$ are $i-v$ fuzzy ideals of $N$.

Definition 3.10. Let $N$ and $N^{\prime}$ be two near-rings, a mapping $f: N \rightarrow N^{\prime}$ is called an $i$-v fuzzy anti-homomorphism if $f(\bar{\mu}+\bar{\sigma})=f(\bar{\mu})+f(\bar{\sigma})$ and $f(\bar{\mu} \bar{\sigma})=f(\bar{\sigma}) f(\bar{\mu})$ where $\bar{\mu}$ and $\bar{\sigma}$ are $i$ - $v$ fuzzy ideals of $N$.

Definition 3.11. Let $f: N \rightarrow N^{\prime}$. An $i-v$ fuzzy subset $\bar{\mu}$ of a near-ring is called $f$ invariant if $f(x)=f(y)$ implies $\bar{\mu}(x)=\bar{\mu}(y), x, y \in N$.

Definition 3.12. $N$ is called a fuzzy multiplication near-ring if for any two $i$ - $v$ fuzzy ideals $\bar{g}$ and $\bar{h}$ of $N$ such that $\bar{g} \subseteq \bar{h}$, there exists a fuzzy ideal $\bar{f}$ of $N$ such that $\bar{g}=\bar{h} \circ \bar{f}$.

Theorem 3.13. If $\bar{h}$ is a prime $i-v$ fuzzy ideal of a fuzzy multiplication near ring $N$ and $\bar{g}$ is any $i-v$ fuzzy ideal of $N$ such that $\bar{h} \subseteq \bar{g}$, then $\bar{h}=\bar{h} \circ \bar{g}$ and $\bar{h}=\bar{g}{ }^{\omega}$ or $\bar{h}=\bar{h}_{\bar{h}} \circ \bar{g}^{\omega}$, where $\bar{g}^{\omega}=\cap\left\{\bar{g}^{i} \mid i \in N \backslash\{0\}\right\}$.

Proof. Since $\bar{h} \subseteq \bar{g}$ and $N$ is an i-v fuzzy multiplication near-ring, there exists an i-v fuzzy ideal $\overline{\bar{k}}$ of $N$ such that $\bar{h}=\bar{g} \circ \bar{k}$. Then since $\bar{h}$ is prime, $\bar{h} \supseteq \bar{k}$. Now $\bar{h}=\bar{g} \circ \bar{k} \subseteq \bar{k}$. Thus $\bar{h}=\bar{k}$ and hence $\bar{h}=\bar{g} \circ \bar{h}$. It now follows that $\bar{h}=\bar{g}{ }^{\omega}$ or $\bar{h}=\bar{h} \circ \bar{g}^{\omega}$.

Theorem 3.14. If $\sqrt{\bar{f}}$ is an i-v prime fuzzy ideal, then $\bar{f}$ is an $i$-v primary.

Proof. Let $\bar{g} \equiv \sqrt{\bar{f}}$. If $\bar{g}=\bar{C}_{N}$, then clearly $\bar{f}$ is an i-v primary. Assume $\bar{g} \neq \bar{C}_{N}$. Suppose that $f$ is not i-v primary. Then there exist i-v fuzzy points $\bar{x}_{r}, \bar{y}_{t}$ such that $\bar{x}_{r} \circ \bar{y}_{t} \subseteq \bar{f}, \bar{x}_{r} \subseteq \bar{g}$, but $\bar{x}_{r} \nsubseteq \bar{f}$ and $\bar{y}_{t}^{n} \nsubseteq \bar{f}$ for all $n>0$. Let $\bar{k}=\bar{f} \cup \bar{g} \circ\left(\bar{x}_{r} \circ \bar{C}_{N}\right)$. Clearly, $\underline{\bar{k}}$ is an i-v fuzzy ideal of $N$. Suppose $\bar{x}_{r} \subseteq \bar{k}$. Then since $\bar{x}_{r} \not \bar{f}, \bar{x}_{r} \subseteq$ $\bar{g} \circ\left(\bar{x}_{r} \circ C_{N}\right)$. Thus $\left(g \bar{\circ}\left(x_{r} \circ C_{N}\right)\right)(x) \geq r$, $\overline{o r} \vee\left\{g(a) \wedge\left(x_{r} \circ C_{N} \overline{(b}\right) \mid x=a b\right\} \geq r$. 
Since $\bar{f}$ has the sup property, $\bar{g}$ also possesses the sup property. Hence there exists $z \in S$ such that $\bar{g}(z) \geq \bar{r}$ and $x=z x s=x z s$. Thus $\bar{f}\left(z^{n}\right) \geq \bar{r}$, for some $n>0$. Now $x=x z^{n} s^{n}$ and since $\bar{f}$ is an i-v fuzzy ideal, $\bar{f}(x)=\bar{f}\left(x z^{n} s^{n}\right) \geq \bar{f}\left(z^{n}\right) \geq \bar{r}$, i.e $\bar{x}_{r} \subseteq \bar{f}$, a contradiction. Hence $\bar{x}_{r} \nsubseteq \bar{k}$. Now, $\bar{k} \cup \bar{x}_{r} \circ \bar{C}_{N} \subseteq \bar{g}$. Thus there exists an i-v fuzzy ideal $\bar{h}$ of $N$ such that $\bar{k} \cup \bar{x}_{r} \circ \bar{C}_{N}=\bar{g} \circ \bar{h}$. Again since $\bar{y}_{t} \nsubseteq \bar{g}, \bar{g} \subseteq \bar{g} \cup \bar{y}_{t} \circ \bar{C}_{N}$. Then by Theorem $3.13 \bar{g}=\bar{g} \circ\left(\bar{g} \cup \bar{y}_{t} \circ \bar{C}_{N}\right)$. Now $\bar{k} \cup \bar{x}_{r} \circ \bar{C}_{N}=\bar{g} \circ \bar{h}=\bar{g} \circ\left(\bar{g} \cup \bar{y}_{t} \circ \bar{C}_{N}\right) \circ \bar{h}=$ $\bar{g} \circ \bar{h} \circ\left(\bar{g} \cup \bar{y}_{t} \circ \bar{C}_{N}\right)$ (since $N$ is commutative) $=\left(\bar{k} \cup \bar{x}_{r} \circ \bar{C}_{N}\right) \circ\left(\bar{g} \cup \bar{y}_{t} \circ \bar{C}_{N}\right) \subseteq \bar{k}$. Hence $\bar{x}_{r} \subseteq \bar{k}$, a contradiction. Therefore, $\bar{f}$ is an i-v primary.

Corollary 3.15. Let $\bar{f}$ be an i-v prime. Then for all positive integers $n, \bar{f}^{n}$ is an $i-v$ primary and its $i-v$ fuzzy radical is $\bar{f}$.

Proof. We first prove that $\sqrt{\bar{f} n}=\bar{f}$ for all $n>0$. If $n=1$, the result is obvious. Let $n>1$. Then $\sqrt{\bar{f} n(x)}=\vee\left\{\bar{f}^{n}\left(x^{m}\right) \mid m>0\right\} \geq \bar{f}^{n}\left(x^{n}\right) \geq \bar{f}(x)$ for all $x \in N$. Since $\bar{f}$ is an i-v prime, $\bar{f}(x)=\sqrt{\bar{f}(x)}=\vee\left\{\bar{f}\left(x^{m}\right) \mid m>0\right\} \geq\left\{\bar{f}^{n}\left(x^{m}\right) \mid m>0\right\}=\sqrt{\bar{f} n(x)}$ for all $x \in N$. Hence $\sqrt{\bar{f} n}=\bar{f}$. The desired result follows from Theorem 3.14

Theorem 3.16. Let $\bar{f}$ be an $i$-v prime fuzzy ideal and $\bar{f}^{n} \neq \bar{f}^{n+1}$ for all $n>0$. Then $\bar{f}^{\omega}$ is an $i-v$ prime fuzzy ideal.

Proof. Let $\bar{x}_{l}, \bar{y}_{m}$ an $i$-v fuzzy points such that $\bar{x}_{l} \nsubseteq \bar{f}^{\omega}$ and $\bar{y}_{m} \nsubseteq \bar{f}^{\omega}$. We show that $\bar{x}_{l} \circ \bar{y}_{m} \nsubseteq \bar{f}^{\omega}$. If $\bar{x}_{l} \nsubseteq \bar{f}, \bar{y}_{m} \nsubseteq \bar{f}$, then since $\bar{f}$ is an $i$-v prime, $\bar{x}_{l} \circ \bar{y}_{m} \nsubseteq \bar{f}$ and so $\bar{x}_{l} \circ \bar{y}_{m} \nsubseteq f^{\omega}$. Suppose $\bar{x}_{l} \subseteq f, \bar{y}_{m} \nsubseteq \bar{f}$. Since $\bar{x}_{l} \nsubseteq f^{\omega}$, there exists a positive integer $p$ such that $\bar{x}_{l} \subseteq \bar{f}^{p}, \bar{x}_{l} \nsubseteq \overline{\bar{f}}^{p+1}$. Since by Corollary $3.15 \bar{f}^{P+1}$ is an $i$-v primary fuzzy ideal with $i$-v fuzzy radical $\bar{f}, \bar{x}_{l} \circ \bar{y}_{m} \not \bar{f}^{p+1}$ and so $\bar{x}_{l} \circ \bar{y}_{m} \not \bar{f}^{\omega}$. The case when $\bar{x}_{l} \not \bar{f}, \bar{y}_{m} \subseteq \bar{f}$ is similar.

Finally, let $\bar{x}_{l}, \bar{y}_{m} \subseteq \bar{f}$. Then there exist positive integers $q, r$ such that $\bar{x}_{l} \subseteq \bar{f}^{q}, \bar{x}_{l} \nsubseteq$ $\bar{f}^{q+1}$ and $\bar{y}_{m} \subseteq \bar{f}^{r}, \bar{y}_{m} \nsubseteq \bar{f}^{r+1}$. Then $\bar{x}_{l} \circ \bar{C}_{N} \subseteq \bar{f}^{q}, \bar{y}_{m} \circ \bar{C}_{N} \subseteq \bar{f}^{r}$. Since $N$ is an $i$-v fuzzy multiplication near ring, there exist $i$-v fuzzy ideals $\bar{g}, h$ of $N$ such that $\underline{x}_{l} \circ \bar{C}_{N}=\bar{f}^{q} \circ \bar{g}, \bar{y}_{m} \circ \bar{C}_{N} \equiv \bar{f}^{r} \circ \bar{h}, \bar{h}, \bar{g} \nsubseteq \bar{f}$. Now, if $\bar{x}_{l} \circ \bar{y}_{m} \subseteq \bar{f}^{q+r+1}$, then $f^{q+r} \circ \bar{h} \circ \bar{g}=\left(f^{q} \circ \bar{g}\right)\left(\bar{f}^{r} \circ \bar{h}\right)=\bar{x}_{l} \circ \bar{y}_{m} \circ \bar{C}_{N} \subseteq \bar{f}^{q+r+1}$. Since $\bar{f}^{q+r+1}$ is an $i$ primary fuzzy ideal with $i$-v fuzzy radical $\bar{f}$ and $\bar{h} \circ \bar{g} \nsubseteq \bar{f}$ (since $\bar{f}$ is an $i$-v prime), $\bar{f}^{q+r} \subseteq \bar{f}^{q+r+1}$. Also $\bar{f}^{q+r} \supseteq \bar{f}^{q+r+1}$. Thus $\bar{f}^{q+r}=\bar{f}^{q+r+1}$, a contradiction. Hence $\bar{x}_{l} \circ \bar{y}_{m} \nsubseteq \bar{f}^{q+r+1}$, i.e., $\bar{x}_{l} \circ \bar{y}_{m} \not \bar{f}^{\omega}$. Thus $\bar{f}^{\omega}$ is an $i$-v prime fuzzy ideal.

Theorem 3.17. If $\bar{f}$ is an $i$-v primary fuzzy ideal, then $\bar{f}=\bar{g}^{n}$ for some positive integer $n$, where $g=\overline{\sqrt{f} \text {. }}$. 
Proof. If $\bar{g}=\bar{C}_{N}$, then $\bar{f}=\bar{C}_{N}$. Assume $\bar{g} \neq \bar{C}_{N}$. Suppose $\bar{f} \subseteq \bar{g}^{\omega}$. Now $\bar{g}$ is an i-v prime fuzzy ideal of $N$ having the sup property. If $\bar{g}^{n} \neq \bar{g}^{n+1}$ for all $n>0$, then by Theorem 3.16, $\bar{g}^{\omega}$ is an i-v prime. Thus $\bar{g}=\sqrt{\bar{f}} \subseteq \sqrt{\bar{g}^{\omega}}=\bar{g}^{\omega}$, a contradiction. Thus either $\bar{f} \subseteq \bar{g}^{n}=\bar{g}^{n+1}$ for some $n>0$, or $\bar{f} \subseteq \bar{g}^{n}$, but $\bar{g} \nsubseteq \bar{g}^{n+1}$ for some $n>0$. In the first case, let $\bar{x}_{r} \subseteq \bar{g}^{n}$. Then $\bar{x}_{r} \circ \bar{C}_{N} \subseteq \bar{g}^{n}$. Also, there exists an i-v fuzzy ideal $\bar{h}$ of $N$ such that $\bar{x}_{r} \circ \bar{C}_{N}=\bar{g}^{n} \circ \bar{h}$. Thus $\bar{x}_{r} \subseteq \bar{x}_{r} \circ \bar{C}_{N}=\bar{g}^{n} \circ \bar{h}=\bar{g}^{n+1} \circ \bar{h}=\bar{g} \circ\left(\bar{x}_{r} \circ \bar{C}_{N}\right)$. Then as in Theorem 3.14, it can be shown that $\bar{x}_{r} \subseteq \bar{f}$. Hence $\bar{f}=\bar{g}^{n}$.

In second case, there exists an i-v fuzzy ideal $\bar{k}$ of $N$ such that $\bar{f}=\bar{g}^{n} \circ \bar{k}, \bar{k} \nsubseteq \bar{g}$. Since $\bar{f}$ is an i-v primary and $\bar{k} \nsubseteq \bar{g}, \bar{g}^{n} \nsubseteq \bar{f}$. Hence $\bar{f}=\bar{g}^{n}$.

Let $\bar{f}, \bar{g}$ be two i-v fuzzy ideals of $N$. Define the fuzzy subset $\bar{f}: \bar{g}$ of $N$ as follows: $\bar{f}: \bar{g}=\cup\{\bar{h} \mid \bar{h}$ is an i-v fuzzy ideal of $N$ such that $\bar{h} \circ \bar{g} \subseteq \bar{f}\}$.

It follows easily that $\bar{f}: \bar{g}$ is an i-v fuzzy ideal of $N$.

Theorem 3.18. If $\bar{f}$ is a proper prime $i-v$ fuzzy ideal and $\bar{g}$ is an $i$-v fuzzy ideal of $N$ such that $\bar{g} \subseteq \bar{f}^{n}$ and $\bar{g} \nsubseteq \bar{f}^{n+1}$ for some $n>0$, then $\bar{f}^{n}=\bar{g}:\left(\bar{y}_{t} \circ \bar{C}_{N}\right)$, where $\bar{y}_{t} \nsubseteq \bar{f}$.

Proof. Since $\bar{g} \subseteq \bar{f}^{n}$, there exists an i-v fuzzy ideal $\bar{h}$ of $N$ such that $\bar{g}=\bar{f}^{n} \circ \bar{h}, \bar{h} \not \bar{f}$. Let $\bar{y}_{\underline{t}} \subseteq \bar{h}, \bar{y}_{t} \not \subset f$. Then $\bar{y}_{t} \circ \bar{C}_{N} \subseteq \bar{h}$ and $\bar{f}^{n} \circ\left(\bar{y}_{t} \circ \bar{C}_{N}\right) \subseteq \bar{f}^{n} \circ \bar{h}=\bar{g}$.

Thus $f^{n} \subseteq g:\left(\bar{y}_{t} \circ \bar{C}_{N}\right)$. Now let $\bar{k}$ be any i-v fuzzy ideal of $N$ such that $\bar{k} \circ\left(\bar{y}_{t} \circ \bar{C}_{N}\right) \subseteq$ $g$. Then $\underline{k} \circ\left(\bar{y}_{t}\right) \circ \bar{C}_{N} \subseteq \bar{f}^{n}$. Now since by Corollary 3.15, $\bar{f}^{n}$ is i-v primary with fuzzy radical $f$ and $\bar{y}_{t} \circ \bar{C}_{N} \nsubseteq \bar{f}, \bar{k} \subseteq \bar{f}^{n}$. Therefore, $\bar{g}:\left(\bar{y}_{t} \circ \bar{C}_{N}\right) \subseteq \bar{f}^{n}$ and hence $\bar{f}^{n}=\bar{g}:\left(\bar{y}_{t} \circ \bar{C}_{N}\right)$.

Proposition 3.19. Let $f: N \rightarrow N^{\prime}$ be a surjective near-ring anti-homomorphism and $\bar{\mu}^{\prime}$ is an $i$-v fuzzy prime ideal of $N^{\prime}$, then $f^{-1}\left(\bar{\mu}^{\prime}\right)$ is an $i$-v fuzzy prime ideal of $N$.

Proof. Let $\bar{\mu}$ and $\bar{\sigma}$ be two i-v fuzzy ideals of $N$ such that

$\bar{\mu} \bar{\sigma} \subseteq f^{-1}\left(\bar{\mu}^{\prime}\right)$

$\Rightarrow f\left(\bar{\mu} \bar{\sigma} \subseteq \bar{\mu}^{\prime}\right)$

$\Rightarrow f(\bar{\sigma}) f(\bar{\mu}) \subseteq \bar{\mu}^{\prime}$

Since $\bar{\mu}^{\prime}$ is an i-v fuzzy prime ideal of $N^{\prime}$

$\Rightarrow f(\bar{\sigma}) \subseteq \bar{\mu}^{\prime}$ or $f(\bar{\mu}) \subseteq \bar{\mu}^{\prime}$

$\Rightarrow \bar{\sigma} \subseteq f^{-1}\left(\bar{\mu}^{\prime}\right)$ or $\bar{\mu} \subseteq f^{-1}\left(\bar{\mu}^{\prime}\right)$

Therefore $f^{-1}\left(\bar{\mu}^{\prime}\right)$ is an i-v fuzzy prime ideal of $N$.

Proposition 3.20. Let $f: N \rightarrow N^{\prime}$ be a surjective near ring anti-homomorphism and $\bar{\mu}^{\prime}$ is an i-v primary fuzzy ideal of $N^{\prime}$, then $f^{-1}\left(\bar{\mu}^{\prime}\right)$ is an i-v primary fuzzy ideal of $N$. 
Proof. Let $\bar{\mu}$ and $\bar{\sigma}$ be two i-v fuzzy ideals of $N$. Such that

$\overline{\mu \sigma} \subseteq \bar{f}^{-1}\left(\bar{\mu}^{\prime}\right)$ and $\bar{\sigma} \nsubseteq f^{-1}\left(\bar{\mu}^{\prime}\right)$

$\Rightarrow f(\overline{\mu \sigma}) \subseteq \bar{\mu}^{\prime}$ and $f(\bar{\sigma}) \nsubseteq \bar{\mu}^{\prime}$

$\Rightarrow f(\bar{\sigma}) f(\bar{\mu}) \subseteq \bar{\mu}^{\prime}$ and $f(\bar{\sigma}) \nsubseteq \bar{\mu}^{\prime}$

$\Rightarrow f(\bar{\mu}) \subseteq \sqrt{\overline{\bar{\mu}}^{\prime}}$ (Since $\bar{\mu}^{\prime}$ is an i-v primary fuzzy ideal)

$\Rightarrow \bar{\mu} \subseteq f^{-1} \sqrt{\left(\bar{\mu}^{\prime}\right)}$

$\Rightarrow \bar{\mu} \subseteq \sqrt{f^{-1}\left(\bar{\mu}^{\prime}\right)}$

Therefore $f^{-1}\left(\bar{\mu}^{\prime}\right)$ is an i-v primary fuzzy ideal of $N$.

Lemma 3.21. If $\bar{\mu}$ is an $i$-v primary fuzzy ideal of a near-ring $N$, then $\sqrt{\bar{\mu}}$ is an $i$ - $v$ prime fuzzy ideal of $N$.

Proof. Let $\bar{\sigma}$ and $\bar{\theta}$ be two i-v fuzzy ideals of $N$ such that $\bar{\sigma} \bar{\theta} \subseteq \sqrt{\bar{\mu}}$ and $\bar{\sigma} \nsubseteq \sqrt{\bar{\mu}}$

$\Rightarrow \bar{\sigma} \bar{\theta} \subseteq \bar{\mu}$ and $\bar{\sigma} \nsubseteq \bar{\mu}$.

Since $\bar{\mu}$ is an i-v primary fuzzy ideal, $\bar{\theta} \subseteq \sqrt{\bar{\mu}}$.

Therefore $\sqrt{\bar{\mu}}$ is an i-v prime fuzzy ideal of $N$.

Proposition 3.22. Let $f: N \rightarrow N^{\prime}$ be a surjective near-ring anti-homomorphism. If $\bar{\mu}$ is an f-invariant $i$ - $v$ fuzzy ideal of $N$ and $\bar{\mu}$, an $i$ - $v$ fuzzy primary ideal of $N$, then $f(\bar{\mu})$ is an $i-v$ fuzzy primary ideal of $N^{\prime}$.

Proof. Let $\bar{\sigma}^{\prime}$ and $\bar{\theta}^{\prime}$ be two i-v fuzzy ideals of $N^{\prime}$ such that $\bar{\sigma}^{\prime} \bar{\theta}^{\prime} \subseteq f(\bar{\mu})$ and $\bar{\sigma}^{\prime} \nsubseteq f(\bar{\mu})$

$\Rightarrow f^{-1}\left(\bar{\sigma}^{\prime} \bar{\theta}^{\prime}\right) \subseteq f^{-1} f(\bar{\mu})$

$\Rightarrow f^{-1}\left(\bar{\sigma}^{\prime} \bar{\theta}^{\prime}\right) \subseteq \bar{\mu}$ and $f^{-1}\left(\bar{\sigma}^{\prime} \subseteq \bar{\mu}\right)$

$f^{-1}\left(\bar{\sigma}^{\prime}\right) f^{-1}\left(\bar{\theta}^{\prime}\right) \subseteq \bar{\mu}$ and $f^{-1}\left(\bar{\sigma}^{\prime}\right) \nsubseteq \bar{\mu}$

$\Rightarrow f^{-1}\left(\bar{\theta}^{\prime}\right) \subseteq \sqrt{\bar{\mu}}$ (Since $\bar{\mu}$ is an i-v primary fuzzy ideal)

$\Rightarrow \bar{\theta}^{\prime} \subseteq \sqrt{f(\bar{\mu})}$.

Therefore $f(\bar{\mu})$ is an i-v fuzzy primary ideal of $N^{\prime}$.

Proposition 3.23. For a surjective near-ring anti-homomorphism $f: N \rightarrow N^{\prime}$, if $\bar{\mu}$ is an $f$-invariant $i$ - $v$ strongly primary fuzzy ideal of $N$ then $f(\bar{\mu})$ is an $i$ - $v$ strongly primary fuzzy ideal of $N^{\prime}$.

Proof. Let $\bar{\mu}$ be an $f$ - invariant i-v strongly primary fuzzy ideal of $N$.

$\Rightarrow \bar{\mu}$ is an i-v primary fuzzy ideal and $(\sqrt{\bar{\mu}})^{n} \subset \bar{\mu}$ for some $n \in N$

$\Rightarrow f(\bar{\mu})$ is an i-v primary fuzzy ideal of $N^{\prime}$.

Since $f(\bar{\mu})$ is an i-v primary fuzzy ideal of $N^{\prime}, \sqrt{f(\bar{\mu})}$ is an i-v prime fuzzy ideal of $N^{\prime}$. (By Lemma 3.21)

Since $\sqrt{f(\bar{\mu})}=\wedge\left\{f(\bar{\sigma}), f(\bar{\sigma})\right.$ is an i-v fuzzy prime ideal of $\left.N^{\prime}, f(\bar{\sigma}) \subseteq f(\bar{\mu})\right\}$. 
Therefore $\left(\sqrt{f(\bar{\mu}}^{n} \subset f(\bar{\mu})\right.$ for some $N \in N$.

Hence $f(\bar{\mu})$ is an i-v strongly primary fuzzy ideal of $N^{\prime}$.

Proposition 3.24. For a surjective near-ring homomorphism $f: N \rightarrow N^{\prime}$, if $\bar{\mu}$ is an $i$-v semi prime fuzzy ideal of $N^{\prime}$, then $f^{-1}\left(\bar{\mu}^{\prime}\right)$ is an $i$-v semi prime fuzzy ideal of $N$.

Proof. Given $\bar{\mu}^{\prime}$ is an i-v semi prime fuzzy ideal of $N^{\prime}$.

$\Rightarrow \bar{\mu}^{\prime}$ is an i-v fuzzy ideal of $N^{\prime}$ and $\bar{\mu}^{2}(x)=\bar{\mu}(x)$ for all $x \in N$.

$\Rightarrow f^{-1}\left(\bar{\mu}^{\prime}\right)$ is an i-v fuzzy ideal of $N$.

Let $f^{-1}\left(\bar{\mu}^{\prime}\right)=\bar{\mu} \Rightarrow\left(\bar{\mu}^{\prime}\right)=f(\bar{\mu})$

Now $\bar{\mu}^{\prime}=\bar{\mu}^{\prime} \bar{\mu}^{\prime}=f(\bar{\mu}) f(\bar{\mu})=f(\bar{\mu} \bar{\mu})=f\left(\bar{\mu}^{2}\right)$

$\Rightarrow \bar{\mu}^{2}=f^{-1}\left(\bar{\mu}^{\prime}\right)=\bar{\mu} \Rightarrow\left[f^{-1}\left(\bar{\mu}^{\prime}\right)\right]^{2}(x)=f^{-1}\left(\bar{\mu}^{\prime}\right)(x)$ for all $x \in N$.

\section{Conclusion}

In this article it is shown that for an i-v primary fuzzy ideal of a near ring $N, \sqrt{\bar{\mu}}$ is an $\mathrm{i}-\mathrm{v}$ prime fuzzy ideal. Further it has been proved for a f-invariant and $\mathrm{i}-\mathrm{v}$ fuzzy primary ideal $\bar{\mu}$ of $N, f(\bar{\mu})$ is also an i-v fuzzy primary ideal.

\section{References}

[1] S. Abou-zaid, on fuzzy sub near-rings and ideals, Fuzzy Sets Systems, 44, (1991), 139-146.

[2] Seung Dongn Kim and HeeSik Kim, On fuzzy ideals of Near-Rings, Bull. Korean math. Soc. 33 (4) (1996), pp. 593-601.

[3] R. Gilmer, Multiplicative ideal theory, Marcel Dekker. New York, 1972. MR0427289(55 No.323)

[4] Jun. Y.B and Kim.K.H, interval valued fuzzy R-subgroups of nearrings, Indian Journal of Pure and, Applied Mathematics, 33 (1) (2002) 71-80.

[5] B. Davvaz, Fuzzy ideals of near-rings with interval valued membership functions, journals of Sciences, Islamic republic of Iran, 12 (2001), No. 2, 171-175.

[6] R. Kumar, Fuzzy subgroups, fuzzy ideals and fuzzy cosets: some properties, Fuzzy Sets and Systems, 48 (1992), 267-274.

[7] T.Kuraoka and N. Kuroki, On fuzzy quotient rings induced by fuzzy ideals, Fuzzy Sets and System, 47 (1992), 381-386. 
International Journal on Recent and Innovation Trends in Computing and Communication

ISSN: 2321-8169 Volume: 9 Issue: 7

DOI: https://doi.org/10.17762/ijritcc.v9i7.5479

Article Received: 30 June 2021 Revised: 08 July 2021 Accepted: 15 July 2021 Publication: 31 July 2021

[8] W. Liu, Operations on fuzzy ideals, Fuzzy Sets and Systems, 8 (1983), 31-41.

[9] D.S. Malik and J.N. Moderson,Fuzzy maximal, radical and primary ideals of a ring, Information Sciences, 53 (1991), 237-250.

[10] T.K. Mukherjee and M.K Sen, On fuzzy ideals of a ring, Fuzzy Sets and Systems, 21 (1987), 99-104.

[11] A. Rosenfeld, Fuzzy groups, Journal of Mathematical Analysis and Application, 35 (1971), 512-517.

[12] Tariq Shah and Muhammad Sayeed, On fuzzy ideals in Rings and Antihomomorphism, International Mathematical Forum, Vol.7(16), (2002), 753-759.

[13] A. Sheikabdullah and K. Jeyaraman, Anti-Homomorphisms in Fuzzy Ideals of Rings, Int.J. Contemp. Math. Sciences, Vol.5, 55 (2010), 2717-2721.

[14] T. Shah and M. Saeed, Fuzzy ideals in Laskerian rings, 65, 1 (2013), 74-81

[15] N. Thillaigovindan, V.Chinnadurai and S. Kadalarasi, Interval valued Fuzzy ideal of Near-rings, J. Fuzzy Math. 23 (2) (2015) 471-484.

[16] L.A. Zadeh, Fuzzy Sets, Inform. and Control, 8 (1965), 338-353. 